\title{
openheart Inception of the 'endocarditis team' is associated with improved survival in patients with infective endocarditis who are managed medically: findings from a before-and-after study
}

Amit Kaura, ${ }^{1,2}$ Jonathan Byrne, ${ }^{1}$ Amanda Fife, ${ }^{3}$ Ranjit Deshpande, ${ }^{1}$ Max Baghai, ${ }^{1}$ Margaret Gunning, ${ }^{1}$ Donald Whitaker, ${ }^{1}$ Mark Monaghan, ${ }^{1}$ Philip A MacCarthy, ${ }^{1}$ Olaf Wendler, ${ }^{1}$ Rafal Dworakowski ${ }^{1}$

\begin{abstract}
- Additional material is published online only. To view please visit the journal online (http://dx.doi.org/10.1136/ openhrt-2017-000699).
\end{abstract}

To cite: Kaura A, Byrne J, Fife A, et al. Inception of the 'endocarditis team' is associated with improved survival in patients with infective endocarditis who are managed medically: findings from a before-and-after study. Open Heart 2017;4:e000699. doi:10.1136/ openhrt-2017-000699

Received 15 August 2017 Revised 12 October 2017 Accepted 7 November 2017

\section{(a) CrossMark}

'Department of Cardiology and Cardiac Surgery, King's College Hospital NHS Foundation Trust, King's College Hospital, London, UK

${ }^{2}$ Department of Cardiovascular Medicine, Imperial College Healthcare NHS Trust, Hammersmith Hospital, London, UK

${ }^{3}$ Department of Microbiology, King's College Hospital NHS Foundation Trust, King's College Hospital, London, UK

Correspondence to Dr Rafal Dworakowski; rdworakowski@gmail.com

\section{ABSTRACT}

Objective Despite improvements in its management, infective endocarditis (IE) is associated with poor survival. The aim of this study was to evaluate the impact of a multidisciplinary endocarditis team (ET), including a cardiologist, microbiologist and a cardiac surgeon, on the outcome of patients with acute IE according to medical or surgical treatment strategies.

Methods We conducted an observational before-andafter study of 196 consecutive patients with definite IE, who were treated at a tertiary reference centre between 2009 and 2015. The study was divided into two periods: period 1, before the formation of the ET $(n=101)$, and period 2, after the formation of the ET $(n=95)$. The role of the ET included regular multidisciplinary team meetings to confirm diagnosis, inform the type and duration of antibiotic therapy and recommend early surgery, when indicated, according to European guidelines.

Results The patient demographics and predisposing conditions for IE were comparable between the two study periods. In the time period following the introduction of the $\mathrm{ET}$, there was a reduction in both the time to commencement of IE-specific antibiotic therapy ( $4.0 \pm 4.0$ days vs $2.5 \pm 3.2$ days; $P=0.004$ ) and the time from suspected IE to surgery (7.8 \pm 7.3 days vs $5.3 \pm 4.2$ days; $P=0.004)$. A 12-month Kaplan-Meier survival for patients managed medically was $42.9 \%$ in the pre-ET period and $66.7 \%$ in the post-ET period $(\mathrm{P}=0.03)$. The involvement of the ET was a significant independent predictor of 1-year survival in patients managed medically ( $\mathrm{HR} 0.24,95 \% \mathrm{Cl} 0.07$ to $0.87 ; \mathrm{P}=0.03$ ).

Conclusions A standardised multidisciplinary team approach may lead to earlier diagnosis of IE, more appropriate individualised management strategies, expedited surgery, where indicated, and improved survival in those patients chosen for medical management, supporting the recent change in guidelines to recommend the use of a multidisciplinary team in the care of patients with IE.

\section{INTRODUCTION}

Despite improvements in its management, infective endocarditis (IE) is associated with

\section{Key questions}

What is already known about this subject?

- Despite improvements in its management, infective endocarditis (IE) is associated with substantial morbidity and poor survival. While current European Society of Cardiology recommendations support the use of a multidisciplinary team approach to improve the management of patients with IE, data in this area are limited.

What does this study add?

- A standardised multidisciplinary team approach may lead to earlier diagnosis of IE, more appropriate individualised management strategies, expedited surgery, where indicated, and improved survival in those patients chosen for medical management.

How might this impact on clinical practice?

- These data provide preliminary evidence to support the recent change in guidelines to recommend the use of a multidisciplinary team in the care of patients with IE.

substantial morbidity and poor survival, with a $14 \%-22 \%$ in-hospital and up to $40 \%$ 1-year mortality. ${ }^{1-3}$ Prompt diagnosis, antibiotic therapy and early surgical intervention, when indicated, have been shown to improve survival. ${ }^{45}$ Despite this, the diagnosis may be delayed $^{6}$ and patients may not receive surgery even when indicated. ${ }^{7}$ Unsurprisingly, guideline non-compliance has been shown to be associated with worse outcome. ${ }^{8}$

To facilitate complex decision-making, a multidisciplinary team (MDT) approach may support the management of patients with IE. While this team-based approach has already been shown to improve clinical care of patients with chronic valvular heart disease,${ }^{9}$ 
especially in the selection of patients with severe aortic stenosis for transcatheter aortic valve implantation procedures ${ }^{10}$ this approach is not yet common for IE despite recent recommendations. The present European Society of Cardiology (ESC) Task Force on IE supports the evaluation and management of patients with IE in reference centres by a specialised MDT consisting of a cardiologist, microbiologist, imaging specialist and a cardiac surgeon (the 'Endocarditis Team' (ET)). ${ }^{11}$ The role of the ET includes regular meetings in order to make prompt diagnoses, choose the type and duration of antibiotic therapy and to facilitate early surgery in those patients with a clear indication. Recently, it has been reported that a teambased approach reduced 1-year mortality from 18.5\% to $8.2 \%$ in a mixed cohort of medically and surgically managed patients with IE. ${ }^{12}$

A formal ET approach to managing patients with IE was introduced at our hospital in 2012. The aim of this study was to evaluate the impact of an MDT management approach on the outcome of patients with IE according to medical or surgical treatment strategies.

\section{METHODS}

\section{Study patients}

We conducted an observational before-and-after study of 196 consecutive patients with definite acute IE, according to the modified Duke criteria, ${ }^{13}$ who were treated at King's College Hospital, a tertiary reference centre. The study was divided into two periods: period 1 , before the formation of the ET (August 2009 to June 2012), and period 2, after the formation of the ET (July 2012 to April 2015).
Demographic, clinical, microbiological, imaging and surgical data were collected prospectively on a computer database. Biochemical data were taken at the time of patient admission. Predisposing conditions included dental or surgical intervention within the past 12 months, intravenous drug abuse, history of IE or heart valve disorders (rheumatic disease, prosthetic valve or valve repair). Vascular and immunological phenomena (glomerulonephritis, Osler nodes, Roth spots and rheumatoid factor) were defined according to recent ESC guidance. ${ }^{11}$ Uncontrolled sepsis was defined as having persistent infection or signs of locally uncontrolled infection as defined by European guidance. ${ }^{11}$ Persistent infection consisted of fever and persistent positive cultures after 7-10 days of appropriate antibiotic treatment.

\section{Multidisciplinary management approach}

Each patient with suspected IE was initially discussed with the cardiology team and underwent a transthoracic echocardiogram (TTE) (figure 1). A transoesophageal echocardiogram (TOE) was performed following the TTE if study quality was poor or if valvular complications were suspected. The patient was also discussed with the microbiology team and blood cultures taken. If there was a high clinical suspicion of IE, the case was then referred to the ET. The ET consisted of two cardiologists, one microbiologist, one cardiac imaging specialist, one cardiac surgeon and an IE specialist nurse coordinator.

New referrals to the ET were discussed immediately after presentation, existing cases at a once-weekly MDT meeting and all cases were reviewed on twice-weekly ward

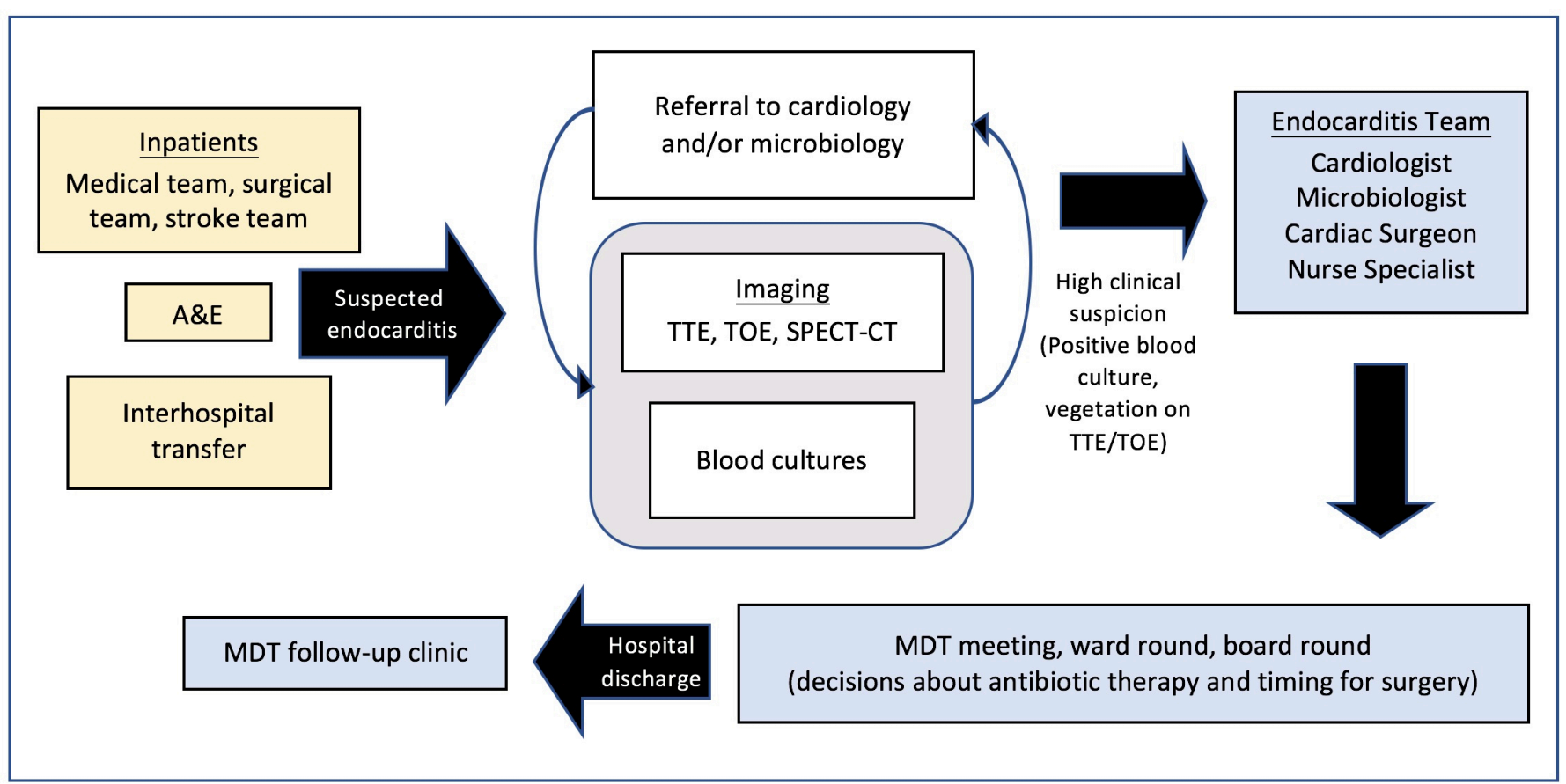

Figure 1 Referral pathway and journey of patients with infective endocarditis. A\&E, accident and emergency; MDT, multidisciplinary team; SPECT, single photon emission CT; TOE, transoesophageal echocardiogram; TTE, transthoracic echocardiogram. 
rounds and board rounds led by a consultant microbiologist and cardiologist. The purpose of this collaborative evaluation was to initially characterise patients as definite, probable or excluded cases of IE, as defined by the modified Duke criteria, ${ }^{13}$ and to subsequently obtain a consensus on the type, duration and mode of antibiotic therapy. Risk of systemic embolisation, progressive heart failure and irreversible structural damage due to severe infection were assessed in accordance with European guidance to determine if there was an indication for early surgery. ${ }^{11}$ All patients requiring surgery were broadly categorised according to indication (embolic, infectious or haemodynamic) and timing (emergency, within 24 hours; urgent, within a few days; elective, after 1-2 weeks of antibiotic therapy). ${ }^{11}$ Time to surgery was defined as the time from suspected IE to the operative procedure. Medical therapy was indicated for those at lower risk, or those patients deemed unfit for surgery. Patients were monitored for medical therapy failure and the development of indications for surgical intervention. The clinical course was evaluated daily by the ward team and reviewed by the ET on a twice-weekly ward round. All patients were followed up at 1, 6 and 12 months following discharge from hospital at an endocarditis clinic run by the ET in the outpatient setting.

\section{Statistical analysis}

Clinical, microbiological and outcome data were compared between the two study periods (pre-ET vs post-ET). We subsequently compared these data according to management strategy (medical, surgical, medical (surgical turndown)). Patients who were managed surgically following an initial period of medical therapy were coded in the surgical strategy group. Categorical data are expressed as absolute and relative frequency and compared using the $\chi^{2}$ test with Yates' correction or Fisher's exact test. Parametric data are presented as mean (SD) and analysed using t-test. Cox proportional hazards regression modelling was used to evaluate the independent contribution of the ET and various clinical parameters on in-hospital and 12-month mortality for all patients and according to each management strategy. Each variable was first entered into a univariate model, and those found to be significant at a level of $\mathrm{P}<0.20$ were then entered into a stepwise forward multivariate model. For mortality analysis, with no loss to follow-up prior to hospital discharge, the $\mathrm{X}^{2}$ test was used to compare in-hospital morality rates between the pre-ET and post-ET groups. With loss to follow-up accounted for as censored events, the Kaplan-Meier method was used to estimate 1-year survival between the pre-ET and post-ET groups, with differences between the curves evaluated with the log-rank statistic. SPSS (V.21.0; SPSS) was used for statistical analysis.

\section{RESULTS}

\section{Demographic characteristics}

There were 196 consecutive patients with definite IE identified during the study period, of which there were 101 and 95 cases in the pre-ET and post-ET periods, respectively. The
Table 1 Demographic, clinical, microbiology and echocardiographic characteristics of patients with definite endocarditis according to study period

\begin{tabular}{llll}
\hline Variable & $\begin{array}{l}\text { Pre-ET } \\
(\mathbf{n}=101)\end{array}$ & $\begin{array}{l}\text { Post-ET } \\
(\mathbf{n}=95)\end{array}$ & P value \\
\hline Age (years) & $56.1 \pm 14.4$ & $57.5 \pm 17.5$ & 0.54 \\
\hline Male & $67(66.3)$ & $73(76.8)$ & 0.12 \\
\hline Diabetes mellitus & $14(13.9)$ & $15(15.8)$ & 0.84 \\
\hline $\begin{array}{l}\text { EuroSCORE II } \\
\text { Biochemical results* }\end{array}$ & $11.7 \pm 10.6$ & $13.1 \pm 10.3$ & 0.35 \\
$\quad$ Serum eGFR (mL/min) & $66.8 \pm 26.1$ & $66.3 \pm 25.1$ & 0.91 \\
\hline Serum haemoglobin $(\mathrm{g} / \mathrm{dL})$ & $91.5 \pm 32.7$ & $89.6 \pm 32.2$ & 0.70 \\
\hline Serum neutrophils $\left(\mathrm{x} 10^{9} / \mathrm{L}\right)$ & $9.1 \pm 5.3$ & $8.2 \pm 5.1$ & 0.23 \\
\hline Serum CRP $(\mathrm{mg} / \mathrm{L})$ & $93.4 \pm 66.1$ & $105.5 \pm 90.5$ & 0.29 \\
\hline
\end{tabular}

Predisposing conditions for IE

\begin{tabular}{|c|c|c|c|}
\hline $\begin{array}{l}\text { Predisposing cardiac } \\
\text { disease† }\end{array}$ & $30(29.7)$ & $26(27.4)$ & 0.75 \\
\hline Intravenous drug abuse & $10(9.9)$ & $9(9.5)$ & 1.00 \\
\hline History of IE & $10(9.9)$ & $9(9.5)$ & 1.00 \\
\hline Intervention (12 months)‡ & $21(20.8)$ & $14(14.7)$ & 0.35 \\
\hline \multicolumn{4}{|l|}{ Localisation of vegetation } \\
\hline AV & $39(38.6)$ & $38(40.0)$ & 0.96 \\
\hline $\mathrm{AV}+$ aortic root & $9(8.9)$ & $8(8.4)$ & \\
\hline MV & $29(28.7)$ & $27(28.4)$ & \\
\hline $\mathrm{AV}+\mathrm{MV}$ & $7(6.9)$ & $8(8.4)$ & \\
\hline TV & $11(10.9)$ & $8(8.4)$ & \\
\hline $\mathrm{AV}+\mathrm{MV}+\mathrm{TV}$ & $2(2)$ & $3(3.2)$ & \\
\hline Pacemaker lead (no valve) & $1(1)$ & $2(2.1)$ & \\
\hline No vegetation identified & $3(3.0)$ & $1(1.1)$ & \\
\hline \multicolumn{4}{|l|}{ Valve type } \\
\hline Native & $71(70.3)$ & $68(71.6)$ & 1.00 \\
\hline Prosthetic & $22(21.8)$ & $22(23.2)$ & \\
\hline Native and prosthetic & $4(4.0)$ & $2(2.1)$ & \\
\hline \multicolumn{4}{|l|}{ Microbiology } \\
\hline Blood culture positive & $82(81.2)$ & 85 (89.5) & 0.11 \\
\hline Viridans group streptococci & $14(13.9)$ & $9(9.5)$ & 0.50 \\
\hline $\begin{array}{l}\text { Streptococcus } \\
\text { gallolyticus }\end{array}$ & $\begin{array}{l}6(5.9) \mathrm{s} 3 \\
(3.2)\end{array}$ & & \\
\hline Staphylococcus aureus & $24(23.8)$ & $27(28.4)$ & \\
\hline Enterococci & $9(8.9)$ & $11(11.6)$ & \\
\hline
\end{tabular}

Data shown as mean \pm SD or number (percentage).

*Admission values.

†Rheumatic disease, prosthetic valve or valve repair. $\ddagger$ Dental or surgical interventions within the past 12 months. $\mathrm{AV}$, aortic valve; CRP, C-reactive protein; eGFR, estimated glomerular filtration rate; ET, endocarditis team; EuroSCORE, European System for Cardiac Operative Risk Evaluation; IE, infective endocarditis; MV, mitral valve; TV, tricuspid valve.

patient demographics and range of conditions predisposing patients to IE were comparable between the two study periods (table 1). The pattern of infection did not alter with 
Table 2 Management and outcome data of patients with definite endocarditis according to study period

\begin{tabular}{llllr}
\hline Variable & $\begin{array}{l}\text { Pre-ET } \\
(\mathbf{n = 1 0 1 )}\end{array}$ & $\begin{array}{l}\text { Post-ET } \\
\text { (n=95) }\end{array}$ & P value & $\begin{array}{c}\text { Post-ET } \\
\text { (Pre-ET involvement) }\end{array}$ \\
\hline Complications & & & & \\
$\quad$ Immunological phenomena & $11(10.9)$ & $11(11.6)$ & 1.00 & $9(81.8)$ \\
\hline Acute stroke & $22(21.8)$ & $19(20.0)$ & 0.86 & $11(57.9)$ \\
\hline Other emboli & $38(37.6)$ & $31(32.6)$ & 0.55 & $16(51.6)$ \\
\hline Congestive cardiac failure & $17(16.8)$ & $15(15.8)$ & 1.00 & $4(26.7)$ \\
\hline Uncontrolled sepsis & $18(17.8)$ & $27(28.4)$ & 0.09 & $8(29.6)$ \\
\hline Valvular abscess & $11(10.9)$ & $13(13.6)$ & 0.66 & $8(61.5)$ \\
\hline Renal failure† & $11(10.9)$ & $10(10.5)$ & 1.00 & $5(50.0)$ \\
\hline Investigations & & & & \\
\hline Time to first TTE (days) & $7.8 \pm 11.6$ & $4.8 \pm 7.9$ & 0.04 & \\
\hline Time to TOE following TTE (days) & $4.9 \pm 4.1$ & $3.5 \pm 3.1$ & 0.02 & \\
\hline Number of patients undergoing TOE & $69(68.3)$ & $77(81.1)$ & 0.05 & \\
\hline Management & & & & \\
\hline Time to IE-specific antibiotics & $4.0 \pm 4.0$ & $2.5 \pm 3.2$ & 0.004 & \\
\hline Duration of stay & $29.2+15.9$ & $23.9 \pm 15.6$ & 0.02 \\
\hline Management strategy & & & \\
\hline Medical & $21(20.8)$ & $24(25.3)$ & 0.55 & \\
\hline Medical (surgical turndown) & $6(5.9)$ & $8(8.4)$ & \\
\hline Surgical & $74(73.3)$ & $63(66.3)$ & \\
\hline Mortality & & & \\
\hline In-hospital & $17(17.5)$ & $12(12.6)$ & 0.42 \\
\hline 1 year & $31(30.7)$ & $22(23.2)$ & 0.25 \\
\hline
\end{tabular}

Data shown as means \pm SD or number (percentage).

${ }^{*}$ Complications occurring before the involvement of the ET during the post-ET period.

†Renal failure requiring renal replacement therapy.

ET, endocarditis team; IE, infective endocarditis; TOE, transoesophageal echocardiogram; TTE, transthoracic echocardiogram.

regard to causative organism or valves infected. No difference in complications, including congestive cardiac failure $(16.8 \%$ vs $15.8 \%, \mathrm{P}=1.00)$ and acute stroke $(21.8 \%$ vs $20.0 \%$, $\mathrm{P}=0.86$ ), was observed between the pre-ET and post-ET study periods (table 2). A distinction was made between the complications occurring before or after the involvement of the ET during the post-ET period (table 2). Just over half of all embolic events were observed prior to the involvement of the ET ( $57 \%$ for acute stroke and $52 \%$ for other emboli). Data on survival at 1 year were not obtainable for three and two patients in the pre-ET and post-ET groups, respectively.

In both time periods, less than $10 \%$ of patients had an appropriate indication for surgery, however, were deemed unfit for surgery and managed medically due to a combination of reasons including a high logistic EuroSCORE, multiple comorbidities, haemodynamic instability due to severe sepsis or congestive cardiac failure, active malignancy or major neurological deficit following a stroke.

\section{Distribution and outcomes in all patients}

The distribution of patients according to management strategy was similar between the two study periods, with approximately three-quarters of patients managed surgically. Indications for surgery were broadly categorised into three groups (online supplementary figure 1). There was a higher proportion of patients who were deemed to have uncontrolled infection as an indication for surgery in the post-ET than in the pre-ET period (pre-ET 30\% vs post-ET $48 \%$; $\mathrm{P}=0.03$ ).

In the time period following the introduction of the ET, there was a significant reduction in both the time from admission to TTE and the time from TTE to TOE, if indicated (table 2). In addition, there was a reduction in the time to commencement of IE-specific antibiotic therapy ( $4.0 \pm 4.0$ days vs $2.5 \pm 3.2$ days; $\mathrm{P}=0.004)$. Overall, there was also a reduction in the mean duration of inpatient stay by $18 \%$ during the post-ET period ( $29.2 \pm 15.9$ days vs $23.9 \pm 15.6$ days; $\mathrm{P}=0.02$ ). While there was a trend towards a reduction in in-hospital mortality ( $17.5 \%$ to $12.6 \%)$ during the post-ET period, this did not reach statistical significance (table 2). A 12-month Kaplan-Meier survival was $69.3 \%$ in the pre-ET period and $76.8 \%$ in the post-ET period $(\mathrm{P}=0.25)$ in all patients. 
Distribution and outcomes according to management strategy

The distribution of patient demographics, clinical characteristics, vegetation localisation on echocardiography and causative organisms were comparable between the pre-ET and post-ET time periods according to management strategy (online supplementary table 1). In comparison to the pre-ET period, there was a trend towards a reduction in both the time from suspected IE to TTE and time to TOE following TTE imaging in the post-ET period, in all three management strategy groups; however, statistical significance was only achieved in the group of patients managed surgically (table 3 ). There was an approximately $15 \%$ increase in the proportion of patients undergoing TOE imaging in the surgical group, prior to surgery, following the involvement of the ET $(\mathrm{P}=0.04)$.

In the pre-ET period, there was a higher proportion of patients with uncontrolled sepsis who were managed medically rather than surgically $(33.3 \%$ vs $10.8 \%$; $\mathrm{P}=0.04)$. The IE sequelae did not alter between the two time periods although the rate of uncontrolled sepsis was significantly higher in those patients who underwent surgery in the post-ET time period $(10.8 \%$ vs $27.0 \%$; $\mathrm{P}=0.03)$.

In the time period following the introduction of the $\mathrm{ET}$, there was a reduction in the time from suspected IE to surgery ( $7.8 \pm 7.3$ days vs $5.3 \pm 4.2$ days; $\mathrm{P}=0.004)$ (table 3 ). The distribution of patients according to indication for timing of surgery, according to European guidance, was similar between the pre-ET and post-ET periods (online supplementary table 2). Overall, there were more patients who satisfied guideline recommendations for timing for surgery in the post-ET period (pre-ET vs postET; $79.7 \%$ vs $92.1 \%$; $\mathrm{P}=0.05)$.

There was an approximately $25 \%$ reduction in the duration of inpatient stay for surgical patients (pre-ET vs postET; $31.4 \pm 14.5$ days vs $24.3 \pm 12.6$ days; $\mathrm{P}=0.003$ ) (table 3 ). While there was a trend towards a reduction in the risk of in-hospital mortality following the inception of the ET in both the surgical and medical groups, this did not reach statistical significance with $\chi^{2}$ analysis. A 12-month Kaplan-Meier survival was $42.9 \%$ in the pre-ET period and $66.7 \%$ in the post-ET period $(\mathrm{P}=0.03)$ for patients managed medically and $81.1 \%$ in the pre-ET period and $85.7 \%$ in the post-ET period $(\mathrm{P}=0.51)$ for patients who underwent surgery (figure 2). The amount and pattern of loss to follow-up, or censored events, was similar between the comparison groups.

\section{Predictors of mortality}

All demographic, clinical and relevant outcome data which were significant in a univariate model (online supplementary tables 3 and 4) were subsequently entered into a multivariate model to determine the predictors of 1-year mortality. For patients managed surgically, greater age, lower estimated glomerular filtration rate, lower haemoglobin and delay in surgery were all associated with a worse 1-year survival in multivariate analysis (table 4). Patients with native valve endocarditis had better survival than those with prosthetic valve endocarditis. A higher serum neutrophil count and an acute stoke were both identified as independent predictors of a worse 1-year mortality in patients managed medically. The involvement of the ET was a significant independent predictor of improved 1-year survival in patients managed medically (HR $0.24,95 \%$ CI 0.07 to 0.87; $\mathrm{P}=0.03$ ) (table 4).

\section{DISCUSSION}

The present study shows a reduction in all-cause mortality over 12 months in patients with IE who were managed medically following the formation of the ET, suggesting an important impact of an MDT approach to patient management. A similar MDT approach has been shown to decrease 1-year mortality from $18.5 \%$ to $8.2 \%$ in all patients with IE, ${ }^{12}$ an effect also seen in other studies. ${ }^{14}$ In contrast to these studies, our analysis specifically identified the impact of the ET approach stratified by management approach. The in-hospital mortality of patients with IE has been shown to vary from $15 \%$ to $30 \%{ }^{215-18}$ In our study, the in-hospital mortality for medically and surgically managed patients is one of the lowest reported, at $8.3 \%$ and $9.5 \%$, respectively, with the in-hospital mortality for all patients at $12.6 \%$.

Although it is difficult to confirm a direct relationship between the lower mortality in medically managed patients and the inception of the ET, intrinsic differences between the two patient populations are unlikely to be responsible for this improved outcome. Patients managed medically during both time periods had similar demographic and clinical characteristics, and also had similar complication rates. The ET involvement in the medically managed cohort was an independent predictor of improved survival in this cohort at 1 year. The improved outcome of medically managed patients with IE observed at our centre could be attributed to the additional clinical experience acquired over time by the cardiologists, microbiologists and cardiac surgeons, and closer collaboration as part of the ET. An initial MDT evaluation allows for the identification of those patients at high risk of serious complications, and consequently those who would benefit from earlier surgical intervention or more aggressive medical/antibiotic management. In this way, the MDT approach offers the potential to alter the clinical course of the disease. ${ }^{4}$

Prior to the inception of the ET, there was a higher proportion of patients with uncontrolled sepsis who were managed medically than surgically. Uncontrolled infection or severe sepsis is the second most frequent cause for early surgical intervention in patients with IE. ${ }^{19}$ In comparison to the pre-ET period, a higher proportion of patients with uncontrolled sepsis underwent surgery after the introduction of the ET. The better survival in the medical therapy group may therefore have resulted from improved patient selection for surgery in the 


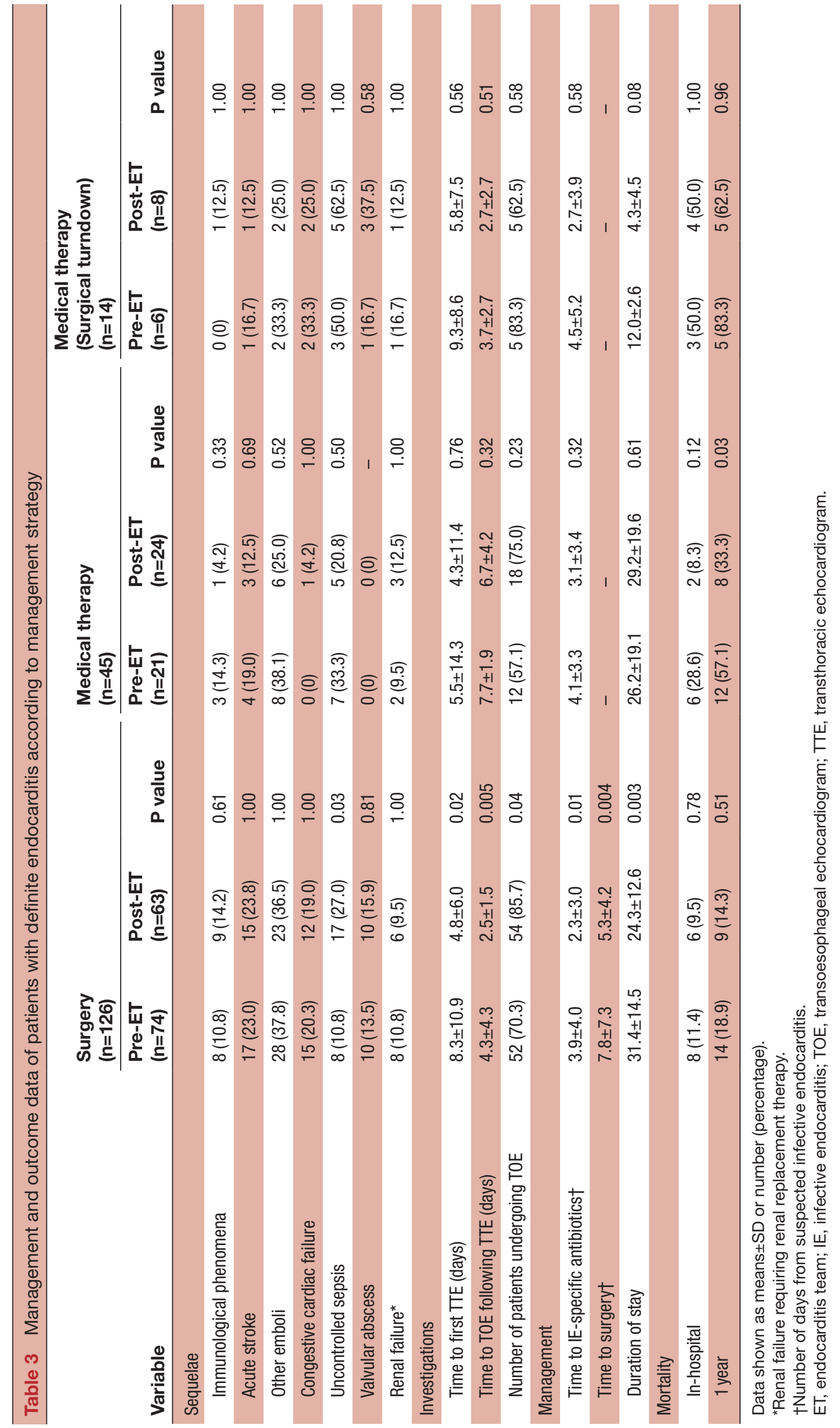




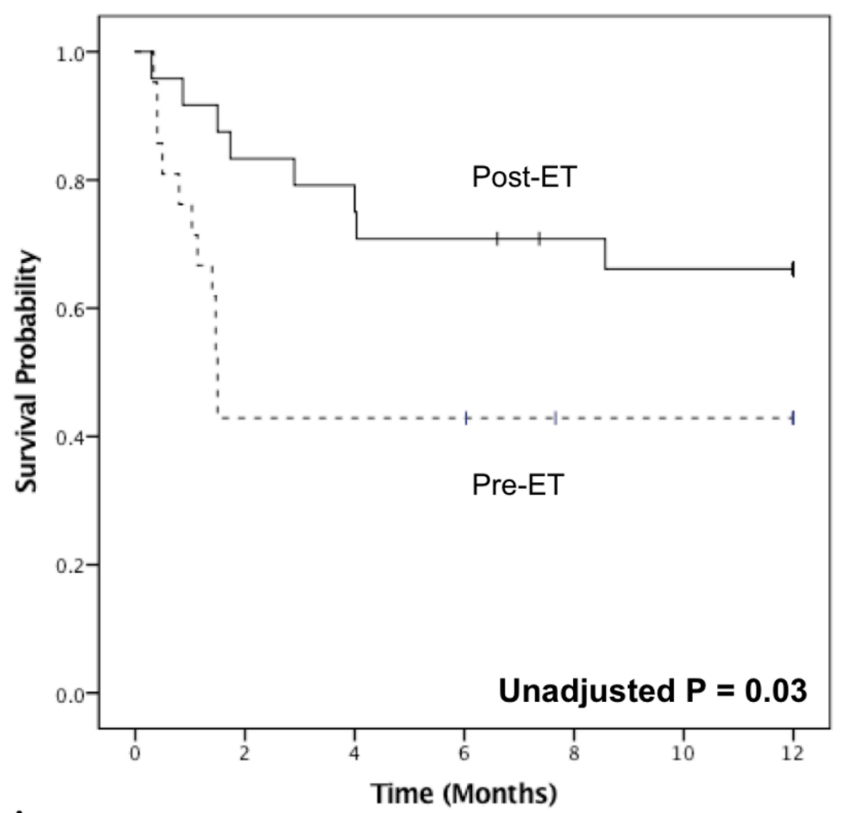

A

Number at Risk

Post- $24 \quad 20$

ET

Pre-

ET

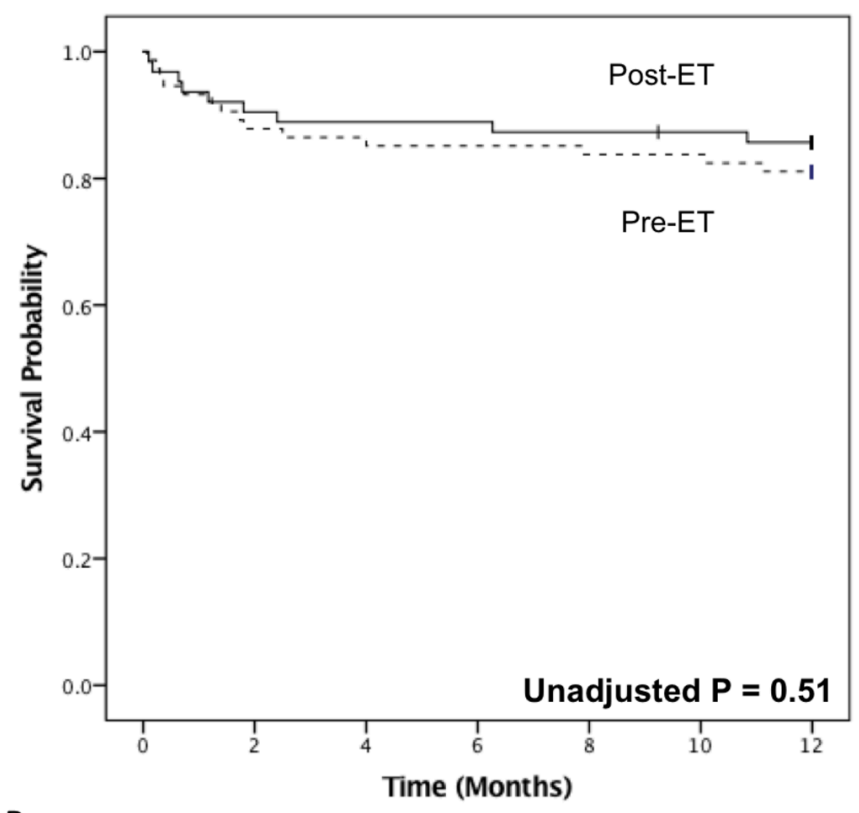

B

Number at Risk

$\begin{array}{llllllll}\begin{array}{l}\text { Post- } \\ \text { ET }\end{array} & 63 & 57 & 56 & 56 & 55 & 54 & 53 \\ \begin{array}{l}\text { Pre- } \\ \text { ET }\end{array} & 74 & 65 & 64 & 63 & 62 & 62 & 60 \\ \end{array}$

Figure 2 Kaplan-Meier survival curves comparing survival between both time periods according to management strategy. Survival in patients with infective endocarditis managed (A) medically and (B) surgically in the periods with (solid line) and without (dotted line) involvement of the endocarditis team (ET). Ticks denote censored events. Differences between the curves evaluated with the log-rank statistic.

post-ET period, while at the same time the mortality in the surgical subgroup was not negatively affected.

In this study, following the introduction of the ET, the rate of surgery declined from $73.3 \%$ to $66.0 \%$. Despite this, the rate of surgery was high in comparison to previous studies (from $40 \%$ to $50 \%$ ). ${ }^{20-22}$ The high rate of surgery in the pre-ET period may reflect a lower threshold for surgical intervention due to less rigid selection policies in comparison to the MDT approach adopted to patient selection for surgery in the post-ET period. Despite guideline recommendations on surgery in IE management, the indications for surgery and its timing are difficult decisions to reach without close collaboration between the cardiologist, cardiothoracic surgeon and microbiologist. ${ }^{11}$ Our in-hospital mortality of $9.5 \%$ following surgery is on the lower end of the $10 \%-20 \%$ mortality, which has been previously reported for patients with acute IE who were managed surgically. ${ }^{23}$ As previously reported, those patients who had a clear indication, but were considered surgically unfit (and were therefore medically managed), had the worst outcomes. ${ }^{24}$

Rapid identification of patients with IE who are at highest risk for death may provide an opportunity to alter the clinical course of the disease process and improve prognosis. We identified a number of clinical predictors of mortality, including patient factors, such as older age, prosthetic valve endocarditis and renal failure, as well as non-cardiac complications, such as acute stroke, findings consistent with other reports in the literature. ${ }^{11} 1725$ Among patients who were medically managed, involvement of the ET was associated with an approximately $75 \%$ lower chance of 1-year mortality. In patients who were managed surgically, a delay in surgery from day of suspected IE was associated with worse in-hospital and 1-year survival. There are data demonstrating that outcome is worse if surgery is delayed, in cases in which surgery is clinically indicated. ${ }^{4626}$

During the post-ET period, there was a $40 \%$ reduction in the time to surgery (from a mean of 8 to 5 days) from the date of suspected IE. While the distribution of patients according to indication for timing of surgery, according to European guidance, was similar between the pre-ET and post-ET periods, deviation from timing recommendations was significantly worse in the pre-ET period. The overall $92 \%$ compliance with timing recommendations for surgery in the post-ET period further highlights the improved communication between the cardiologists and cardiothoracic surgeons. 
Table 4 Multivariate analysis of mortality according to management strategy

\begin{tabular}{|c|c|c|c|c|c|c|}
\hline \multirow[b]{2}{*}{ Variable } & \multicolumn{3}{|c|}{ In-hospital mortality } & \multicolumn{3}{|c|}{ 1-year mortality } \\
\hline & HR & $95 \% \mathrm{Cl}$ & $P$ value & HR & $95 \% \mathrm{Cl}$ & P value \\
\hline \multicolumn{7}{|l|}{ Surgical patients } \\
\hline Age (years) & & & & 1.04 & 1.00 to 1.08 & 0.03 \\
\hline EuroSCORE II & 1.00 & 1.00 to 1.01 & 0.28 & - & - & - \\
\hline Serum eGFR (mL/min)* & & & & 0.98 & 0.96 to 0.99 & 0.005 \\
\hline Serum haemoglobin $(\mathrm{g} / \mathrm{dL})^{\star}$ & & & & 0.99 & 0.98 to 1.00 & 0.05 \\
\hline Serum neutrophils $\left(\times 10^{9} / \mathrm{L}\right)^{*}$ & & & & 1.02 & 0.92 to 1.12 & 0.74 \\
\hline Serum CRP $(\mathrm{mg} / \mathrm{L})^{*}$ & 1.01 & 1.0 to 1.02 & 0.07 & 1.01 & 1.00 to 1.01 & 0.12 \\
\hline Predisposing cardiac disease $\dagger$ & & & & 1.81 & 0.31 to 10.45 & 0.51 \\
\hline Intervention (12 months)‡ & & & & 0.30 & 0.07 to 1.34 & 0.12 \\
\hline Native valve & & & & 0.36 & 0.15 to 0.90 & 0.03 \\
\hline Positive blood culture & & & & 0.74 & 0.25 to 2.23 & 0.60 \\
\hline Valvular abscess & 2.30 & 0.54 to 9.78 & 0.26 & 1.46 & 0.50 to 4.26 & 0.49 \\
\hline Renal failure§ & 4.01 & 1.01 to 16.07 & 0.05 & 1.36 & 0.27 to 6.95 & 0.71 \\
\hline Left-sided IE & 0.46 & 0.09 to 2.38 & 0.35 & & & \\
\hline Time to surgery & 1.13 & 1.06 to 1.20 & 0.03 & 1.11 & 1.05 to 1.17 & 0.001 \\
\hline \multicolumn{7}{|l|}{ Medical patients } \\
\hline Age (years) & & & & 1.02 & 0.98 to 1.07 & 0.32 \\
\hline Serum neutrophils $\left(\times 10^{9} / \mathrm{L}\right)^{*}$ & 1.08 & 0.90 to 1.31 & & 1.21 & 1.04 to 1.41 & 0.02 \\
\hline Positive blood culture & 0.11 & 0.02 to 0.64 & & 0.44 & 0.11 to 1.73 & 0.24 \\
\hline Acute stroke & 2.32 & 0.35 to 15.49 & 0.38 & 12.30 & 3.04 to 49.72 & $<0.0001$ \\
\hline Duration of inpatient stay & & & & 1.00 & 0.99 to 1.01 & 0.89 \\
\hline ET & & & & 0.24 & 0.07 to 0.87 & 0.03 \\
\hline
\end{tabular}

All demographic, clinical and outcome data (apart from mortality) were first entered into a univariate model (online supplementary tables 1 and 2), and those found to be significant at a level of $\mathrm{P}<0.20$ were then entered into a stepwise forward multivariate model.

${ }^{*}$ Admission values.

†Rheumatic disease, prosthetic valve or valve repair.

‡Dental or surgical interventions within the past 12 months.

$\S$ Renal failure requiring renal replacement therapy.

CRP, C-reactive protein; eGFR, estimated glomerular filtration rate; ET, endocarditis team; EuroSCORE, European System for Cardiac Operative Risk Evaluation; IE, infective endocarditis.

Relative to the pre-ET period, there was a significant rise in the proportion of patients undergoing TOE imaging, in the post-ET period, to aid with the diagnosis of mechanical complications such as abscess or valve dehiscence. In addition, we observed a significant reduction in the time to TOE imaging, following a TTE, from 5 days to 3.5 days. While the average time to TOE seems relatively long, at 3.5 days in the post-ET group, the waiting time was as short as 2.5 days in those who went on to have surgery, reflecting appropriate risk stratification of requests for TOE imaging for those suspected of having a potential indication for surgery. Initial MDT evaluation was therefore pivotal in both identifying those patients who may benefit from surgery, and facilitating appropriate investigations to define those with potential surgical indications.

Although there was a non-significant trend towards a reduction in mortality in the surgical cohort following the introduction of the ET, these results did not reach statistical significance. This is most likely explained by the fact that our surgical approach to acute IE has not changed during the study period. The goal during surgery is to radically excise the infected tissue and reconstruct cardiac morphology, including repairing or replacing the affected valve(s) with replacement of the aortic root, if necessary. ${ }^{27} 28$

Importantly, there was a reduction in the mean duration of length of hospital stay by $20 \%$ during the post-ET period from approximately 30 days to 24 days. This may somewhat be due to a significant reduction in the time to surgery in the surgical cohort, with an associated reduction in the total duration of patient stay. While there may be financial as well as clinical benefits with the MDT approach, there is a need to consider the costs of administering, preparing for and attending MDT meetings when evaluating cost-effectiveness. An MDT approach has been shown to be cost-effective in delivering inpatient and ambulatory care to patients with heart failure. ${ }^{2930}$ Further 
studies aimed at understanding the cost-effectiveness of the MDT approach to IE care are required.

\section{Study limitations}

This study has several potential limitations. First, it is a single-centre observational study and at risk of the inherent bias of this type of study. Second, although we included data on 196 consecutive patients with IE with 53 deaths at 12 months, our population is relatively small, therefore increasing the risk of a type II error and of an underestimation of further potential benefits derived from the ET. Third, although our study favourably reflects data from a relatively short time period in comparison to previous studies, variations in epidemiology and temporal improvements in clinical care may have affected the results. Fourth, given the observational design of our study, it is not possible to establish the cause of death for all patients as well as ascertain a direct causal relationship between the reduction in mortality in patients with IE managed medically with the introduction of the ET. Given the number of comparisons performed there is an inherent risk of type 1 error.

\section{CONCLUSIONS}

A standardised MDT approach may lead to earlier diagnosis of IE, more appropriate individualised management strategies, expedited surgery, where indicated, and improved survival in those patients chosen for medical management. Furthermore, the care from the ET was identified as a predictor of survival in patients managed medically. While surgery should be liberally considered according to guideline recommendations, medical management in appropriately selected patients is feasible with excellent outcomes if patients are under close observation. These data provide preliminary evidence to support the recent change in guidelines to recommend the use of an MDT in the care of patients with IE.

Contributors Study conception and design: RD, JB, AK. Acquisition of data: AK, $\mathrm{RD}, \mathrm{JB}, \mathrm{AF}, \mathrm{MG}$. Analysis and interpretation of data: AK, RD, JB,OW, MM, PAMacC. Drafting of manuscript: AK, RD, JB, OW, MM, PAMacC, AF, RD, MB, MG, DW. Critical revision: $A K, R D$.

Funding This work was supported in part by a National Institute for Health Research Biomedical Research Centre award to Guy's \& St Thomas' Hospital and King's College London in partnership with King's College Hospital.

Competing interests None declared.

Patient consent Detail has been removed from this case description/these case descriptions to ensure anonymity. The editors and reviewers have seen the detailed information available and are satisfied that the information backs up the case the authors are making.

Ethics approval King's College Hospital, Cardiovascular Division Review Board.

Provenance and peer review Not commissioned; externally peer reviewed.

Data sharing statement All data are provided in full in the Results section of this paper. Additional data supporting this study are provided as supplementary information accompanying this paper.

Open Access This is an Open Access article distributed in accordance with the Creative Commons Attribution Non Commercial (CC BY-NC 4.0) license, which permits others to distribute, remix, adapt, build upon this work non-commercially, and license their derivative works on different terms, provided the original work is properly cited and the use is non-commercial. See: http://creativecommons.org/ licenses/by-nc/4.0/ (c) Article author(s) (or their employer(s) unless otherwise stated in the text of the article) 2017. All rights reserved. No commercial use is permitted unless otherwise expressly granted.

\section{REFERENCES}

1. Thuny F, Giorgi R, Habachi R, et al. Excess mortality and morbidity in patients surviving infective endocarditis. Am Heart $J$ 2012;164:94-101.

2. Murdoch DR, Corey GR, Hoen B, et al. Clinical presentation, etiology, and outcome of infective endocarditis in the 21st century: the International Collaboration on Endocarditis-Prospective Cohor Study. Arch Intern Med 2009;169:463-73.

3. Kaura A, Dworakowska D, Dworakowski R. Infective endocarditis Cinderella in cardiology. Kardiol Pol 2017;75:965-74.

4. Thuny F, Beurtheret S, Mancini J, et al. The timing of surgery influences mortality and morbidity in adults with severe complicated infective endocarditis: a propensity analysis. Eur Heart $J$ 2011;32:2027-33.

5. Kang DH, Kim YJ, Kim SH, et al. Early surgery versus conventional treatment for infective endocarditis. N Engl J Med 2012;366:2466-73.

6. Delahaye F, Rial MO, de Gevigney G, et al. A critical appraisal of the quality of the management of infective endocarditis. J Am Coll Cardiol 1999;33:788-93.

7. Kiefer T, Park L, Tribouilloy C, et al. Association between valvular surgery and mortality among patients with infective endocarditis complicated by heart failure. JAMA 2011;306:2239-47.

8. González De Molina M, Fernández-Guerrero JC, Azpitarte J. [Infectious endocarditis: degree of discordance between clinical guidelines recommendations and clinical practice]. Rev Esp Cardiol 2002;55:793-800.

9. Lancellotti P, Rosenhek R, Pibarot P, et al. ESC Working group on valvular heart disease position paper-heart valve clinics: organization, structure, and experiences. Eur Heart $J$ 2013;34:1597-606.

10. Vahanian A, Alfieri O, Andreotti F, et al. Guidelines on the management of valvular heart disease (version 2012). Eur Heart J 2012;33:2451-96.

11. Habib G, Lancellotti P, Antunes MJ, et al. 2015 ESC Guidelines for the management of infective endocarditis: the task force for the management of infective endocarditis of the European Society of Cardiology (ESC). Endorsed by: European Association for CardioThoracic Surgery (EACTS), the European Association of Nuclear Medicine (EANM). Eur Heart J 2015;36:3075-128.

12. Botelho-Nevers E, Thuny F, Casalta JP, et al. Dramatic reduction in infective endocarditis-related mortality with a management-based approach. Arch Intern Med 2009;169:1290-8.

13. Li JS, Sexton DJ, Mick N, et al. Proposed modifications to the Duke criteria for the diagnosis of infective endocarditis. Clin Infect Dis 2000;30:633-8.

14. Chirillo F, Scotton P, Rocco F, et al. Impact of a multidisciplinary management strategy on the outcome of patients with native valve infective endocarditis. Am J Cardiol 2013;112:1171-6.

15. Leone S, Ravasio V, Durante-Mangoni E, et al. Epidemiology, characteristics, and outcome of infective endocarditis in Italy: the Italian Study on Endocarditis. Infection 2012;40:527-35.

16. Nadji G, Rusinaru D, Rémadi JP, et al. Heart failure in left-sided native valve infective endocarditis: characteristics, prognosis, and results of surgical treatment. Eur J Heart Fail 2009;11:668-75.

17. Olmos C, Vilacosta I, Fernández C, et al. Contemporary epidemiology and prognosis of septic shock in infective endocarditis. Eur Heart J 2013;34:1999-2006.

18. García-Cabrera E, Fernández-Hidalgo N, Almirante B, et al. Neurological complications of infective endocarditis: risk factors, outcome, and impact of cardiac surgery: a multicenter observational study. Circulation 2013;127:2272-84.

19. Tornos P, lung B, Permanyer-Miralda G, et al. Infective endocarditis in Europe: lessons from the Euro heart survey. Heart 2005;91:571-5.

20. Castillo JC, Anguita MP, Ramírez A, et al. Long term outcome of infective endocarditis in patients who were not drug addicts: a 10 year study. Heart 2000;83:525-30.

21. Gelsomino S, Maessen JG, van der Veen F, et al. Emergency surgery for native mitral valve endocarditis: the impact of septic and cardiogenic shock. Ann Thorac Surg 2012;93:1469-76.

22. Wang A, Athan E, Pappas PA, et al. Contemporary clinical profile and outcome of prosthetic valve endocarditis. JAMA 2007;297:1354-61.

23. Thuny F, Grisoli D, Collart F, et al. Management of infective endocarditis: challenges and perspectives. Lancet 2012;379:965-75. 
24. Mirabel M, Sonneville R, Hajage D, et al. Long-term outcomes and cardiac surgery in critically ill patients with infective endocarditis. Eur Heart J 2014;35:1195-204.

25. Revilla A, López J, Vilacosta I, et al. Clinical and prognostic profile of patients with infective endocarditis who need urgent surgery. Eur Heart J 2007;28:65-71.

26. Muhlestein JB. Infective endocarditis: how well are we managing our patients? J Am Coll Cardiol 1999;33:794-5.

27. Silaschi M, Nicou N, Deshpande R, et al. Complicated infective aortic endocarditis: comparison of different surgical strategies. Interact Cardiovasc Thorac Surg 2017;25:343-9.
28. de Kerchove L, Vanoverschelde JL, Poncelet A, et al. Reconstructive surgery in active mitral valve endocarditis: feasibility, safety and durability. Eur J Cardiothorac Surg 2007;31:592-9.

29. Wijeysundera HC, Machado M, Wang X, et al. Cost-effectiveness of specialized multidisciplinary heart failure clinics in Ontario, Canada. Value Health 2010;13:915-21.

30. Capomolla S, Febo O, Ceresa M, et al. Cost/utility ratio in chronic heart failure: comparison between heart failure management program delivered by day-hospital and usual care. J Am Coll Cardiol 2002;40:1259-66. 